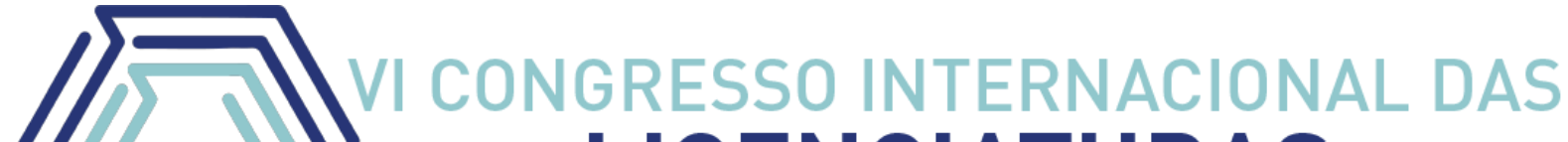 LICENCIATURAS COINTER - PDVL 2019
}

\section{HOROSCOPO QUÍMICO: UMA PROPOSTA DIDÁTICA NO CONTEÚDO DE TABELA PERIÓDICA}

\author{
HORÓSCOPO QUÍMICO: UNA PROPUESTA DE ENSEÑANZA EN EL \\ CONTENIDO DE LA TABLA PERIÓDICA
}

\section{CHEMICAL HOROSCOPE: A TEACHING PROPOSAL IN THE PERIODIC TABLE CONTENT}

\author{
Apresentação: Comunicação Oral \\ Flávio José de Abreu Moura ${ }^{1}$; Wilson Antonio da Silva ${ }^{2}$; Palloma Joyce de Aguiar ${ }^{3}$ Gabriela \\ Rejane Silva de Medeiros ${ }^{4}$; Ronaldo Dionísio da Silva ${ }^{5}$
}

DOI: $\underline{\text { https://doi.org/10.31692/2358-9728.VICOINTERPDVL.2019.0115 }}$

\section{Resumo}

O Ensino de Química é visto pela maioria dos estudantes como de difícil compreensão por se tratar de conceitos abstratos e sem sentido. Talvez essa apatia, seja recorrente do ensino tradicional (re)produzido pelos professores, no qual, o enfoque é na memorização e repetição de conceitos, tratando o aluno como depósito de conhecimento. Refletindo sobre isso, faz-se necessário pensar em estratégias que ajudem os estudantes numa melhor visualização e compreensão desses conceitos. Para isso,o presente trabalho mostracomo a estratégia didática"Horoscopo Químico" pode contribuir de forma lúdica e atrativa no aprendizado e no ensino do conteúdo de tabela periódica.Justifica-se esta ação pelo fato de que muitos estudantes associam que essa ferramenta precisa ser memorizada por completo, não tendo nenhuma utilidade que faça sentido para os mesmos. A pesquisa possui uma abordagem qualitativa, sendo realizada na EREM Guiomar Krause Gonçalves que é uma escola parceira do Programa Residência Pedagógica, em uma turma com 23 estudantes do primeiro ano do Ensino Médio na cidade de Vitória de Santo Antão - PE. Para o desenvolvimento da atividade, utilizamos como base teórica metodológica, o Ciclo da Experiência Kellyana (CEK), que segue cinco passos básicos: Antecipação, Investimento, Encontro, Confirmação ou Desconfirmação e Revisão Construtiva. A partir da vivência do CEK, analise aos questionamentos e debates com o público, coletamos resultados bastante positivos,

\footnotetext{
${ }^{1}$ Licenciatura em Química, Instituto Federal de Educação Ciência e Tecnologia de Pernambuco - CampusVitória, flavio.jose33@hotmail.com

${ }^{2}$ Licenciatura em Química, Instituto Federal de Educação Ciência e Tecnologia de Pernambuco - CampusVitória, wilson.antonio98@hotmail.com

${ }^{3}$ Licenciatura em Química, Instituto Federal de Educação Ciência e Tecnologia de Pernambuco - CampusVitória, palloma joyce aguiar@hotmail.com

${ }^{4}$ Licenciatura em Química, Instituto Federal de Educação Ciência e Tecnologia de Pernambuco - CampusVitória, madeirosgabriela32@yahoo.com.br

${ }^{5}$ Doutor em Química, Universidade Federal de Pernambuco, ronaldo.dionisio@vitoria.ifpe.edu.br
} 
percebendo a evolução dos estudantes após a realização da aula com a atividade Lúdica. Eles foram capazes argumentar de forma correta e cientifica sobre os questionamentos feitos, além de confirmarem e/ou desconfirmar as hipóteses iniciais. Desse modo, consideramos que metodologias inovadores, criativas e lúdicas, acrescentam e muitono aprendizado e na vida dos estudantes.

Palavras-Chave:Atividade Lúdica, Propriedades Periódicas, Ensino de Química, Elemento Químico.

\section{Resumen}

La mayoría de losestudiantesconsideran que laenseñanza de la química es difícil de entender porque se trata de conceptos abstractos y sin sentido. Quizásestaapatía es recurrente de laenseñanza tradicional (re) producida por los maestros, enlacualel foco está enlamemorización y larepetición de conceptos, tratando al estudiante como un depósito de conocimiento. Al reflexionar sobre esto, es necesario pensar enestrategias que ayuden a losestudiantes a visualizar y comprendermejorestos conceptos. Para esto, el presente trabajomuestracómolaestrategiadidáctica "Horóscopo químico" puede contribuir de una manera lúdica y atractivaenelaprendizaje y laenseñanzadelcontenido de la tabla periódica. Esta acción se justifica por elhecho de que muchosestudiantesasocian que estaherramientanecesita ser memorizada por completo, sintenerningún uso que tenga sentido para ellos. La investigacióntieneun enfoque cualitativo, que se lleva a cabo en EREM Guiomar Krause Gonçalves, que es una escuelaasociadadel Programa de Residencia Pedagógica, en una clasecon 23 estudiantes de secundaria de primer añoenlaciudad de Vitória de Santo Antão - PE. Para eldesarrollo de laactividad, utilizamos como base metodológica teórica, elKellyana Experience Cycle (CEK), que sigue cinco pasos básicos: anticipación, inversión, encuentro, confirmación o desconfirmación y revisiónconstructiva. A partir de la experiencia de CEK, analizarlas preguntas y los debates conel público, obtuvimos resultados muy positivos, notando laevolución de losestudiantesdespués de laclaseconlaactividad lúdica. Pudieron discutir correcta y científicamente sobre las preguntas formuladas y confirmar y / o desconfirmarlashipótesisiniciales. Por lo tanto, consideramos que lasmetodologíasinnovadoras, creativas y lúdicas agreganmucho al aprendizaje y la vida de losestudiantes.

Palabras Clave:Actividad de juego, propiedades periódicas, enseñanza de la química, elemento químico.

\footnotetext{
Abstract

Chemistry teaching is seen by most students as difficult to understand because it is abstract and meaningless concepts. Perhaps this apathy is recurrent from the traditional teaching (re) produced by teachers, in which the focus is on memorization and repetition of concepts, treating the student as a repository of knowledge. Reflecting on this, it is necessary to think of strategies that help students better visualize and understand these concepts. For this, the present work shows how the didactic strategy "Chemical Horoscope" can contribute in a playful and attractive way in the learning and teaching of the periodic table content. This action is justified by the fact that many students associate that this tool needs to be memorized completely, having no use that makes sense to them. The research has a qualitative approach, being conducted at the EREM Guiomar Krause Gonçalves which is a partner school of the Pedagogical Residence Program, in a class with 23 first year high school students in the city
} 
of Vitória de Santo Antão - PE. For the development of the activity, we use as the theoretical methodological basis, the Kellyana Experience Cycle (CEK), which follows five basic steps: Anticipation, Investment, Encounter, Confirmation or Disconfirmation and Constructive Review. From the experience of CEK, analyze the questions and debates with the public, we collected very positive results, noticing the evolution of students after the class with the playful activity. They were able to argue correctly and scientifically about the questions asked, and confirm and / or disconfirm the initial hypotheses. Thus, we consider that innovative, creative and playful methodologies add a lot to the learning and life of students.

Keywords: Play Activity, Periodic Properties, Chemistry Teaching, Chemical Element.

\section{Introdução}

O ensino das Ciências da Natureza, principalmente o de química, ainda gera entre os estudantes uma sensação de desconforto em função das dificuldades de aprendizagem de alguns conceitos abstratos, decorrente do processo de ensino aprendizagem. Comumente, tal ensino ainda é realizado de maneira tradicional, levando em consideração apenas a memorização, apresentando os conceitos de forma descontextualizada e não interdisciplinar. Quando o ensino é realizado dessa maneira os estudantes não encontram sentido ao que estão aprendendo, gerando um grande desinteresse pela matéria, bem como dificuldades de aprender e de relacionar o conteúdo estudado ao cotidiano, mesmo a química estando presente na realidade (ROCHA, 2016).

Quando os estudantes encontram um sentindo ao conteúdo que estás sendo estudado, eles se sentem atraídos e pertencentes.Segundo Nunes \&Adorn (2010) afirmam que, diferentemente do modelo tradicional (que visa a memorização como base do aprendizado, levando em consideração os alunos como meros armazenadores de conhecimento), é necessário possibilitar aos estudantes a compreensão contextualizada dos conteúdos químicos, para que seja possível julgá-los como fundamentos teórico-práticos.

O crescente desinteresse dos alunos em relação aos estudos, em específico relacionado a disciplina de Química, bem como a presença de salas de aulas cada vez mais superlotadas e heterogêneas, forçou a busca por metodologias de ensino-aprendizagem mais atraentes (PAULETTI, 2017).

O conteúdo de tabela periódica é visto por boa parte dos estudantes como algo difícil, chato e que não tem finalidade alguma. Desse modo, é viável o uso de ferramentas que auxiliem na compreensão por parte dos alunos. Santos, Alves e Castro (2010) afirmam que a utilização de metodologias inovadoras de ensino que envolvam arte, modelos e/ou jogos mostram-se eficazes ao proporcionar que o estudante seja ativo, contribuindo numa 
construção diária de conhecimentos.Essas metodologias exigem que o aluno saia de sua zona de conforto, passando a ser um sujeito ativo e participativodas atividades propostas, argumentando e mostrando seu ponto de vista sobre determinado conteúdo.

Quando abordada de forma lúdica, a químicase torna bem mais interessante e atrativa para os estudantes, permitindo uma maior interação entre conhecimento-professor-aluno, trazendo contribuições no processo de ensino e aprendizagem.

De acordo com Souza e colaboradores, as atividades lúdicas propiciam no aluno a indagação de seu raciocínio, sua reflexão e consequentemente a construção do seu conhecimento. Além de promover a construção do conhecimento cognitivo, químico, social e psicomotor o que leva a assimilar mais facilmente o assunto abordado. (Souza et al, 2018)

Todavia, há um grande desinteresse por parte de alguns docentes em elaborar estratégias diferenciadas, por muitas vezes a escola não disponibilizar dos recursos necessários para a construção de algum material ou pela falta de tempo devido à grande demanda de carga horária. Há também alguns docentes que já não acreditam mais em seus estudantes e não enxergam com bons olhos essas estratégias.

A prática docente deve ser baseada na intenção de reforçar a capacidade crítica do educando, sua curiosidade e sua insubmissão, abrindo espaço para a dúvida e o questionamento que possam surgir a partir da atividade vivenciada. Desse modo, a atuação do professor em sala de aula é de suma importância para auxiliar no processo de ensino e aprendizagem, facilitando a compreensão dos conteúdos e fortalecendo a perspectiva do saber como instrumento de compreensão e intervenção no mundo (FREIRE, 1996).

O presente trabalho justifica-se pela grande dificuldade que os alunos possuem acerca do conteúdoTabela Periódica, visto que o mesmo se faz essencial para o aprendizado e entendimento da química de modo geral. Desse modo, o trabalho tem como objetivo proporcionar aos estudantes um aprendizado lúdico acerca do conteúdo de "Tabela Periódica”, em uma turma com 23 estudantes do $1^{\circ}$ Ano do Ensino Médio utilizando o Ciclo da Experiência Kellyana (CEK) como referencial metodológico, no qual, o professor agiu como mediador da construção do conhecimento de seus estudantes, buscando analisar os saberes prévios e empíricos acerca do conteúdo. Além, de procurar descrever as contribuições proporcionadas aos estudantes a partir da atividade lúdica "Horoscopo Químico" e se a ferramenta foi realmente eficaz para o ensino.

Para o desenvolvimento das atividades utilizamos como recursos didáticos, imagens 
de tabelas periódicas e o "Horoscopo Químico". Para a realização da explanação do conteúdo utilizamos o quadro branco e piloto.

\section{Fundamentação Teórica}

O ensino de ciências nas escolas brasileiras ainda é na sua grande maioria conduzido de forma tradicional, tendo como base a simples memorização e repetição de fórmulas e/ou cálculos, deixando de lado a ideia da correlação com a realidade dos estudantes.Por esses motivos os estudantes não encontram sentido ao que estão aprendendo (SOUSA, 2018). Pensando dessa forma, Fialho (2011) salienta que a falta de interesse do aluno é derivada da forma impositiva que o professor retrata ao repassar conteúdos, impingindo regras rígidas e tratando os conteúdos de forma fria e distante.

Quando se trata do ensino de Química, tem sido cada vez mais exigido novas propostas criativas e inovadoras de metodologias, visando contribuir para a melhoria do processo de ensino aprendizagem dos estudantes (LIMA, 2011). Pode-se destacar também que o ensino dessa disciplina, bem como de outras que utilizam de cálculos matemáticos, tem um problema sério da antipatia de boa parcela dos estudantes (FERNANDES, 2013).

Os Parâmetros Curriculares Nacionais para o Ensino Médio (PCNs), alegam que os estudantes precisam desenvolver competências e habilidades que sejamcapazes de correlacionara Química com o mundo real, possibilitando uma visão mais ampla do conhecimento, contribuindo com a construção da cidadania (BRASIL, 2000). Alegando ainda, que o estudante deve "reconhecer aspectos químicos relevantes na interação individual e coletiva do ser humano com o ambiente" (BRASIL, p. 39, 2000).

Pensando dessa forma, "o conhecimento dos princípios de organização e uso da Tabela Periódica dos Elementos - TPE faz parte dessa cultura científica e, portanto, o aprendizado sobre esse tema é essencial quando buscamos o letramento científico dos estudantes" (FIALHO; VIANNA; RICARDO; SCHMITT, p, 267, 2018).

De acordo com Leach (2018) a tabela periódica dos elementos é a base da química, visto que a ciência que envolve o estudo da matéria e suas transformações, amplia-se a partir dessa ferramenta, na qual os elementos químicos são organizados.

Desse modo, podemos entender:

"a relevância do ensino e aprendizagem da tabela periódica, porém o estudo desta temática precisa ser realizado de maneira dinâmica e interessante para que o estudante tenha interesse em aprender. Além disso, é fundamental que 
o professor busque novas estratégias de ensino e crie oportunidades para que o estudante construa seu próprio conhecimento sobre o assunto abordado." (FIALHO; VIANNA; RICARDO; SCHMITT, p, 268, 2018)

Para esse processo faz-se indispensável proporcionar condições e habilidades aos estudantes, necessárias para participar de momentos de debates, questionamentos, reflexões, exposição e confronto de ideias. (DA SILVA, 2019). Para isso utilizamos o ciclo da experiência Kellyana, onde a reconstrução da realidade vivida está relacionada à ideia de que, se uma pessoa não aprende, ela não viveu a experiência (VIANA, 2014).

George Kelly (1963),defende uma teoria psicológica que considera as pessoas como construtoras de seus próprios conhecimentos, através de um processo denominado Alternativismo Construtivo (BASTOS, 1992). Essa teoria é denominada de Teoria dos Constructos Pessoais (TCP) onde possui 11 corolários, sendo um deles o Corolário da Experiência onde diz que "O sistema de construção de uma pessoa varia à medida que ela constrói sucessivamente, réplicas de eventos" (KELLY, 1963, p. 72). Nesse caso, quando a realidade não está de acordo com a réplica, a pessoa modifica seu sistema de construção. Esse processo de reconstrução está relacionado à ideia de Kelly sobre aprendizagem. Pois, para ele, a aprendizagem não é algo que acontece apenas nas escolas, ou em algumas ocasiões especificas, mas um processo diretamente ligado à vivência de uma experiência.

A aprendizagem ocorre a partir de 5 etapas: antecipação, investimento, encontro, confirmação ou desconfirmação e revisão construtiva. O ciclo se inicia na etapa da Antecipação, momento em que o estudante é convidado para participar de um determinado evento e usa os construtos que possui para construir ideias relevantes sobre um determinado conceito e tenta antecipá-lo. Na segunda etapa, o Investimento, momento de melhorar a construção feita na etapa anterior, através da inclusão de novos elementos. É o momento de preparação para o encontro com o evento, através de leituras, conversas e reflexões. Em seguida o estudante é engajado na etapa do Encontro, é o momento onde os estudantes se deparam com o evento. É a ocasião mais esperada pelos alunos onde o professor utiliza de algum artificio didático como jogos, softwares e experimentos, o que conduz à quarta etapa, a Confirmação ou Desconfirmação. Nessa etapa, a pessoa confirma ou refuta suas hipóteses iniciais presentes na etapa da Antecipação e ampliadas na etapa do Investimento, a partir da vivência no evento de suas teorias pessoais. E fechando o CEK, a etapa da Revisão Construtiva, em que o indivíduo revê seus construtos anteriores, consolida seus conhecimentos e repensa toda a situação. 


\section{Metodologia}

A pesquisa apresenta natureza Qualitativa e caracterizada como estudo de caso, pois foram analisados processos que não podem ser quantificados ou medidos experimentalmente em termos de quantidade, volume, intensidade ou frequência (DENZIN E LINCOLN, 2006).

Utilizamos como instrumento de pesquisa: o quadro branco, piloto e imagens de tabelas periódicas para explanação do conteúdo, também utilizamos de uma atividade lúdica para fazer referência as características e propriedades da tabela periódica. Como procedimento da pesquisa utilizamos a vivência do CEK (Ciclo da Experiência Kellyana) os quais estão descritos abaixo.

1. Antecipação:Nesta etapa realizamos alguns questionamentos, envolvendo o conteúdo de tabela periódica, para sabermos o quanto eles conheciam sobre o assunto e através do que foi relatado tentamos no decorrer da aula despertar o interesse deles pela atividade.

\section{P1: Para que serve a tabela periódica?}

P2: Átomo e elemento químico são a mesma coisa?

\section{P3: O que são famílias e períodos?}

\section{P4: Como é organizada a tabela periódica?}

2. Investimento: Nesse momento ocorreu a explanação do conteúdo através de uma aula expositiva utilizando o quadro e piloto, levando em consideração as hipóteses iniciais levantadas pelos estudantes. Durante esse momento de explanação, foi oferecido um espaço para que os estudantes pudessem fazer questionamentos sobre o assunto e sanar suas dúvidas. Mostramos a evolução da tabela periódica, como e para que a utilizar, suas propriedades e como ela é organizada hoje em dia.

3. Encontro: Esse foi o momento mais esperado pelos estudantes, onde cada aluno construiu uma espécie de Horoscopo Químico, baseado nos símbolos dos elementos químicos que estavam presentes nos seus nomes próprios. 
3.1.Primeiro pedimos para que eles escrevessem seu primeiro nome pessoal ou como eles preferem ser chamados em uma folha de papel.

3.2.Em seguida, com a ajuda de uma tabela periódica, pedimos que eles identificassem os símbolos dos elementos químicos que estavam presentes em seus nomes e indicassem a qual família eles pertenciam. Fizemos um exemplo no quadro do que deveria ser feito (Figura 1).

Figura 1.Nome com a identificação dos símbolos dos elementos.

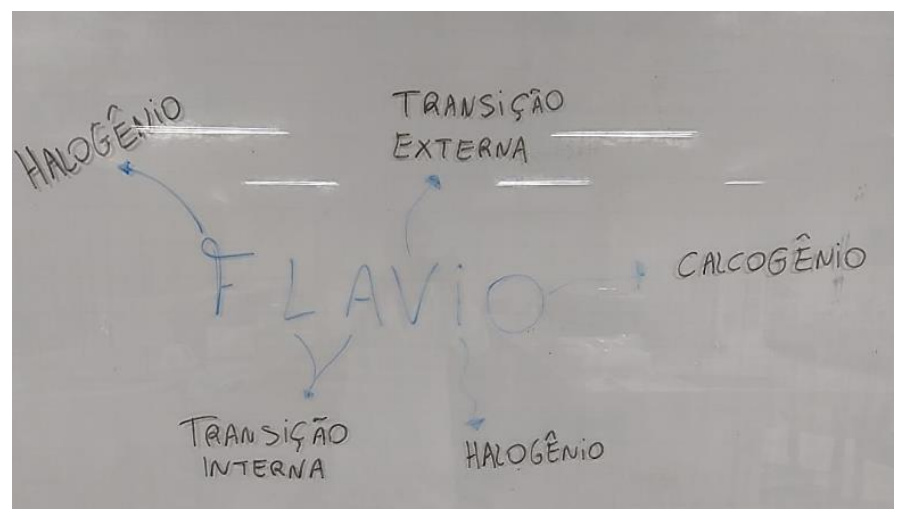

Fonte: Própria.

3.3.Após todos os estudantes realizarem essa etapa, apresentamos a eles uma tabela (Tabela 1) onde relacionava as propriedades ou características de cada família da tabela periódica com adjetivos pessoais, fazendo uma analogia a um horoscopo.

Tabela 1.Tabela do Horoscopo Químico.

\begin{tabular}{|l|l|}
\hline \multicolumn{2}{|c|}{ HOROSCOPO QUÍMICO } \\
\hline Hidrogênio & Solitário \\
\hline Metais Alcalinos & Explosivo (Reativo \\
\hline Metais Alcalinos Terrosos & Frágil (Mole) \\
\hline Família do Boro & Bruto (Solido em temperatura ambiente) \\
\hline Família do Carbono & Poligamia (4 Ligações) \\
\hline Família do Nitrogênio & Sufocante (Pnicogênios) \\
\hline Calcogênio & Interesseiro (Forma composto com o Cu) \\
\hline Halogênio & Atraente (Eletronegatividade) \\
\hline Gases Nobres & Estável \\
\hline
\end{tabular}




\begin{tabular}{|l|l|}
\hline Metais de Transição Externa & Influenciável (Maleável) \\
\hline Metais de Transição Interna & Invejoso (semelhanças entre os elementos) \\
\hline Fonte: Própria.
\end{tabular}

A tabela mostra propriedades e/ou características de cada família da tabela periódica, mostrando ainda um adjetivo humano que se assemelhe com tal característica.

4. Confirmação ou Desconfirmação: Nesta etapa fizemos alguns questionamentos parecidos, com os utilizados na etapa antecipação, porém, um pouco mais elaboradas visto que eles vivenciaram as etapas do investimento e o encontro.

P5: Porque a tabela periódica não é organizada por ordem de massa atômica?

P6: O que as famílias da tabela periódica representam?

P7: Qual a diferença entre átomo e elemento Químico?

P8: O que é eletronegatividade? E qual o elemento mais eletronegativo?

A partir das respostas dos estudantes fizemos uma análise afim de observar se houve confirmação ou desconfirmação dos conhecimentos prévios e/ou ganho de "novos" conhecimentos científicos.

5. Revisão Construtiva:Em formato de roda de conversa, pedimos para que os estudantes relatassem sobre a experiencia vivida, alegando se gostaram da metodologia utilizada, se conseguiram compreender, baseados nos seguintes tópicos:
a) A aula foi produtiva?
b) A aula foi divertida/dinâmica?
c) Conseguiu compreender o conteúdo?
d) Com aulas nesse estilo, conseguimos chamar sua atenção para aprender o conteúdo?
e) Acha que seria preciso algumas mudanças para a aula ficar melhor, ter sido mais produtiva? Se sim, quais sugestões você nos daria?
f) A metodologia utilizada facilitou o aprendizado? 
E por fim realizamos um auto avaliação sobre todo o processo, buscando refletir sobre a metodologia utilizada.

\section{Resultados e Discussão}

A seguir, elencamos algumas respostas e argumentosque representam ou resumem os questionamentos feitos durante a primeira etapa do Ciclo, aAntecipação.

No primeiro questionamento P1, observamos que os estudantes conhecem um pouco sobre tabela periódica, decorrente de aulas anteriores, porém, há uma confusão entre elemento Químico e átomo:

Estudante A: "Para guardar os átomos"

Estudante B: "Não sei, é aquela tabela lá, dos elementos"

Estudante C: "Para organizar os átomos"

Estudante D: "Para mostrar as o que tem nos átomos"

Após recebermos essa mistura de conceitos, automaticamente fizemos um questionamento sobre o assunto P2. Toda a turma, concordou que elemento químico seria um sinônimo de átomo. Essas confusões conceituais foram sendo sanadas ao decorrer das etapas do ciclo.

No terceiro questionamento $\mathbf{P 3}$,percebemos um melhor entendimento a respeito de famílias e períodos:

Estudante A: "É como os elementos estão distribuídos na tabela."

Estudante B: "Família são as linhas de cima para baixo e períodos as linhas deitadas"

Estudante C: "Nas famílias os elementos são parecidos e nos períodos não"

Estudante D: "É uma forma de agrupar os elementos"

Mesmo apresentando uma linguagem informal, os estudantes demonstraram ter um conhecimento prévio a respeito do assunto.

Quando questionamos o que era determinante para a organização periódica dos elementos, muitos dos estudantes não souberam responder ou responderam de forma errada.

Estudante A: "pelo tamanho do átomo" 
Estudante B: "Não sei"

Estudante C: "Pela massa atômica"

Estudante D: "Não sei”

A partir dos dados coletados, conseguimos identificar e determinar quais conceitos seriam dado mais enfoque.

Em seguida partimos para o Investimento,onde realizamos a explanação dos conteúdos. Começamos com a evolução histórica da tabela periódica, destacando as influencias na tabela atual.Mostramos também como extrair informações da tabela atual, dando enfoque nas propriedades periódicas e nas características de cada família.Citamos exemplos de elementos encontrados facilmente no dia-a-dia.

No encontro, realizamos a atividade lúdica "Horoscopo Químico", onde conseguimos perceber uma forte interação aluno-aluno e professor-aluno, diante do que estava sedo proposto. Ao mesmo tempo que os estudantes se divertiam com os adjetivos relacionados aos seus nomes, eles aprendiam que aquele símbolo representava um elemento de uma determinada família, onde essa família possuía característica análoga ao adjetivo que lhe foi concebido.

Os estudantes foram ativos durante a atividade, sempre participando através de questionamentos e levantando hipóteses com base na explanação realizada anteriormente. Demos todo o suporte na construção dos nomes, ajudando na localização dos símbolos e fazendo algumas adaptações quando necessário.

Na Confirmação ou Desconfirmação, realizamos questionamentos parecidos com os da antecipação, e percebemos uma evolução dos conhecimentos científicos dos estudantes e também a confirmação e desconfirmação de algumas hipóteses.

Na P5 todos os estudantes apresentaram respostas satisfatórias e condizentes, mostrando um avanço no aprendizado dos estudantes:

Estudante A: "A organização por massa atômica é limitada"

Estudante B: "Alguns elementos possuem massas atômicas semelhantes"

Estudante C: "A massa atômica não é a identidade dos elementos"

Estudante D: “Os cientistas descobriram que não era a melhor escolha, pois alguns elementos possuíam massas parecidas" 
As respostas da P6foi um surpreendente consenso dos estudantes, alegando que as "famílias são as colunas verticais", onde cada uma "apresentam características semelhantes" entre os elementos pertencentes.

Quando retomamos a pergunta P7, a qual havia gerado um conflito de ideias inicialmente, percebemos a desconfirmação de hipóteses de uma parte considerável dos estudantes:

Estudante A: "O átomo é apenas um e o elemento é um mol de átomos"

Estudante B: "Elemento é a junção de vários átomos iguais"

Estudante C: "Um monte de átomos dá origem aos elementos"

Estudante D: "Os elementos são aqueles que tem na tabela periódica... um monte de átomo é que forma um elemento químico"

Por fim, questionamos a cerca de uma das propriedades periódicas, a eletronegatividade. Muitos associaram aos nomes dos colegas que apresentavam a letra F, visto que essa letra representa o elemento mais eletronegativo, o Flúor.

Estudante A: "O poder de atração dos elétrons... Flúor"

Estudante B: "O flúor é o que mais atrai elétrons."

Estudante C: "O elemento atrai os elétrons pra ele"

Estudante D: "Eu lembrei do meu nome que tem F, que seria o Flúor... e ele puxa os elétrons mais pra perto dele"

Diante dessas respostas, percebemos a contribuição da atividade, onde os alunos relacionaram os adjetivos dado as famílias com as propriedades reais deles.Os símbolos que tinham em seus próprios nomes ajudaram a lembrar e fazer essa relação.

Por fim, chagamos na ultima etapa a Revisão construtiva. Aqui fizemos alguns questionamentos e reflexões afim de entendermos como os estudantes avaliaram essa estratégia didática. Diante dos argumentos dos estudantes, ficou claro o entusiasmo dos mesmos, alegando que "a aula foi muito interessante..." principalmente no "...momento da construção do horoscopo", dessa forma eles "conseguiram aprender fazendo relações com 
adjetivos pessoais."

Os alunos sugeriram que deveria ser feito alguma relação aos signos, já que era um horoscopo, porém, afirmaram que isso não atrapalhou em nada o aprendizado deles. Acreditamos que o trabalho tem uma grande relevância, visto que as partes envolventes saíram satisfeitas.

\section{Conclusões}

A utilização de metodologiasatrativas e inovadorasé essencial para consolidação de determinados conceitos químicos, principalmente daqueles que são vistos como chatos e/ou difíceis. A tabela periódica dos elementos químicos por exemplo, é um desses conteúdos, onde os alunos a enxergam como algo que deve ser memorizado e não consultado.

Tomando isso como base, realizamos a atividades Lúdica "Horoscopo Químico" com o intuito de desmistificar essa ideia erronia que os estudantes tem sobre tabela periódica.Para isso utilizamosde investigações dos conhecimentos prévios e empíricos dos estudantes, os quais foram retomados durante as outras etapas do Ciclo da Experiencia Kellyana.

A partir da vivência do CEK e os dados coletados, a atividade lúdica "Horoscopo Químico" teve bastante aceitação do público, ficando evidenciado pela participação efetiva dos mesmos.Muitas vezes, os estudantes não se envolvem no conteúdo pela metodologia utilizada pelo professor.Dessa forma, é necessário que o corpo docente se mobilize e saia da zona de conforto, procurando atividades diferenciadas e atrativas para seus alunos. A realização de atividades como essas, permitem a autonomia dos discentes, na busca de soluções aos problemas que os cerca, além de exercitar e demonstrar noções básicas de Química.

\section{Referências}

BASTOS, H. F. B. N. Changing teachers' practice: towards a constructivist methodology of physics teaching, Inglaterra, 1992. Tese (Doutorado em Física), UnversityofSurrey. Não publicado.

BRASIL. Parâmetros curriculares nacionais do ensino médio. Ciências da natureza,
matemática $\quad$ e
<http://portal.mec.gov.br/seb/arquivos/pdf/ciencian.pdf> acessado em Setembro de 2018.

DA SILVA, Taiza de Souza Gusmões. Ensino de ciências e experimentação nos anos iniciais: da teoria a prática. PRÓ-DISCENTE, v. 25, n. 1, 2019.

DENZIN, N. K. e LINCOLN, Y. S. Introdução: a disciplina e a prática da pesquisa qualitativa. In: DENZIN, N. K. e LINCOLN, Y. S. (Orgs.). O planejamento da pesquisa qualitativa: 
teorias e abordagens. 2. ed. p. 15-41. Porto Alegre: Artmed, 2006.

FERNANDES, M. L. M.; O Ensino de Química e o Cotidiano, $1^{\text {a }}$ ed., InterSaberes: Curitiba, 2013.

FIALHO, N. N.; Jogos no Ensino de Química e Biologia, 2a ed., Ibpex: Curitiba, 2011.

FIALHO, N. N.; VIANNA F., RICARDO P.; SCHMITT, M. R. O uso de mapas conceituais no ensino da tabela periódica: um relato de experiência vivenciado no PIBID. Química Nova na Escola, v. 40, n. 4, p. 267-275, 2018.

FREIRE, P. Pedagogia da autonomia: saberes necessários à prática educativa. São Paulo: Paz e Terra, 1996.

KELLY, A.G. A theory of personality: the psychology of personal constructs. New York: W.W. Norton, 1963.

LEACH, M. R. ChemogenesisWebBook. Disponível em http:// www.metasynthesis.com/webbook.html, acessado em setembro de 2018.

LIMA, E. C. et al. Uso de jogos lúdicos como auxílio para o ensino de química. Revista Eletrônica Educação em Foco, v. 3, 2011.

NUNES, A. S.; Adorni, D.S. O ensino de química nas escolas da rede pública de ensino fundamental e médio do município de Itapetinga-BA: O olhar dos alunos. In: Encontro Dialógico Transdisciplinar - Enditrans, 2010, Vitória da Conquista, BA. -Educação e conhecimento científico, 2010.

PAULETTI, Fabiana. Entraves ao ensino de química: apontando meios para potencializar este ensino. Revista Areté| Revista Amazônica de Ensino de Ciências, v. 5, n. 8, p. 98-107, 2017.

ROCHA, J. S.; VASCONCELOS, T. C. Dificuldades de aprendizagem no ensino de química: algumas reflexões. XVIII Encontro Nacional de Ensino de Química (XVIII ENEQ), p. 110, 2016.

SANTOS, V. F.; ALVES, B. H. P.; CASTRO, L. M. de. ELABORAÇÃO E APLICAÇÃO DE JOGOS DIDÁTICOS NAS AULAS DE QUÍMICA NO ENSINO FUNDAMENTAL E MÉDIO. Seminário de Iniciação Científica do IFG, n. 4, Goiás, 2010.

SOUZA, E. C. et al. O Lúdico como Estratégia Didática para o Ensino de Química no $1^{\circ}$ Ano do Ensino Médio.Revista Virtual de Química, v. 10, 2018.

SOUZA, G. A. P. et al. Elaboração de Materiais Didáticos: Possibilidades na Formação de Professores de Química. Revista Debates em Ensino de Química, Recife, v. 4, n. 1, p. 47-58, 2018.

VIANA, K. S. L. Avaliação da experiência: uma perspectiva de avaliação para o ensino das 
ciências da natureza. 2014. 202 f. Tese (Doutorado em Ensino das Ciências) - Universidade Federal de Pernambuco, Recife, 2014. 